ISSN: 2386-3919

ISSN electrónico: 2386-3927

DOI: http://dx.doi.org/10.14201/et2015332191211

\title{
EXIGENCIAS DIDÁCTICAS EN DISEÑO DIDÁCTICO DE TAREAS PARA EL DESARROLLO DE LAS HABILIDADES INVESTIGATIVAS
}

\section{Instructional Exigencies in Instructional Design Task for the Development of Research Skills}

\section{Enseignement Exigences en tâche de conception pédagogique pour le développement des compétences de recherche}

Odiel Estrada MolinA*, Sahara María Blanco HernándeZ*** y

Febe Ángel CIUDAD RICARDO***

Universidad de las Ciencias Informáticas de Cuba

* Departamento de Ciencias Sociales y Humanidades de la Facultad, 6. Correo-e: oestrada@uci.cu

* Centro de Innovación y Calidad de la Educación (CICE) de la Universidad de las Ciencias Informáticas de Cuba

Recibido: 02-05-2014; Aceptado: 31-01-2015; Publicado: 30-10-2015

BIBLID [2386-3927 (2015) 33, 2; 191-211]

Ref. Bibl. ODIEL ESTRADA MOLINA, SAHARA MARÍA BLANCO HERNÁNDEZ y FEBE ÁNGEL CIUDAD RICARDO. Exigencias didácticas en diseño didáctico de tareas para el desarrollo de las habilidades investigativas. Enseñanza \& Teaching, 33, 2-2015, 191-211. http://dx.doi.org/10.14201/et2015332191211

RESUMEN: Este artículo aborda la formación de las habilidades de la competencia investigativa asociada a la industria del software desde la relación UniversidadIndustria. Se fundamenta la deficiencia didáctica existente en las investigaciones 
ODIEL ESTRADA, SAHARA MARÍA BLANCO Y FEBE ÁNGEL CIUDAD EXIGENCIAS DIDÁCTICAS EN DISEÑO DIDÁCTICO DE TAREAS PARA EL DESARROLLO DE LAS HABILIDADES INVESTIGATIVAS

realizadas en el área de la didáctica para afrontar el desarrollo de estas habilidades en estudiantes de carreras asociadas a la informática en cuanto al desarrollo de software industrial. Para brindar solución a esta deficiencia, se realizó una sistematización teórica acerca de la formación de las habilidades investigativas, se determinaron las particularidades didácticas del desarrollo de software industrial y se determinaron exigencias didácticas a tener en cuenta para el desarrollo de estas habilidades en este tipo de contexto. Se presentan como resultados: (1) exigencias didácticas a tener en cuenta por los y las profesionales de la industria del software en el diseño didáctico de tareas según las particularidades de este tipo de industria y en función del desarrollo de las habilidades de la competencia investigativa; (2) acciones para contribuir a su desarrollo desde la relación Universidad-Industria; y (3) valoraciones exploratorias de 120 estudiantes y 40 profesionales de la industria según el nivel de aceptación que poseen los y las estudiantes sobre el proceso de formación que reciben de los y las profesionales de la industria cubana de software; nivel de conocimiento y habilidades en el diseño didáctico de tareas por parte de los y las profesionales de la industria; el nivel de aceptación acerca de la propuesta presentada en esta investigación; y el nivel de compromiso de los y las profesionales ante la formación del estudiante. Para el desarrollo de este tipo de competencia se deben tener en cuenta los distintos niveles de comunicación que se evidencian en el proceso de desarrollo de software industrial.

Palabras clave: didáctica; habilidades de la competencia investigativa; industria del software; ingeniería informática; habilidades investigativas.

SUMMARY: This paper addresses the formation of the research competence associated with the software industry from the University-Industry. Deficiency in the existing educational research in the area of teaching to address the development of research competence in students of careers related to computing in the development of industrial software is presented. To provide a solution to this educational deficiency, a theoretical systematization of the formation of the research was performed competition, didactic particularities industrial software development was determined. Its presents, didactic requirements for industry professionals in the instructional tasks design for the development of the research skill; actions to contribute to its development from the University-Industry. It's presents exploratory evaluations of 120 students and 40 industry professionals as the level of acceptance that students about his training in the industry, level of knowledge and skills in instructional design task by industry professionals, the level of acceptance about the proposal presented in this research, and the level of professional commitment to student training. The development of this competence is determinate for the different levels of communication that is evident in the industrial software.

Key words: didactics; computer engineering; research competence; research skills; software industry. 
RÉSUMÉ: Cet article traite de la formation des compétences en matière de compétences de recherche associée à l'industrie du logiciel de l'Université-Industrie. Irrégularité dans la recherche en éducation existant dans le domaine de l'enseignement pour répondre au développement de ces étudiants à des carrières informatiques associés dans le développement de logiciels industriels est basée. Pour apporter une solution à cette carence éducative, une systématisation théorique sur les compétences en recherche de formation a été menée particularités didactique développement de logiciels industriels a été déterminée. Les résultats sont présentés comme suit: (1) d'envisager l'enseignement par des professionnels de l'industrie dans la conception pédagogique des tâches en fonction des spécificités de cette industrie et en fonction de l'évolution des compétences de recherche des besoins de compétences; (2) actions pour contribuer à son développement de l'Université-Industrie; et (3) les évaluations exploratoires de 120 étudiants et 40 professionnels de l'industrie comme: le niveau d'acceptation que les étudiants ont à peu près le processus de réception des professionnels de la formation industrie du logiciel de Cuba; niveau de connaissances et de compétences en enseignement tâche de conception par des professionnels de l'industrie; sur le niveau d'acceptation de la proposition présentée dans cette recherche; et le niveau d'engagement professionnel à la formation des étudiants. Pour le développement de ce type de compétition est à prendre en compte les différents niveaux de communication qui est évident dans le processus de développement de logiciels industriels.

Mots clés: enseignement; industrie du logiciel; ingénierie informatique; la compétence des compétences de recherche; les compétences de recherche.

\section{INTRODUCCIÓN}

La formación de las habilidades de la competencia investigativa es un tema que se ha estado abordando en los últimos años pues permite al estudiante universitario brindar solución a problemas profesionales desde una concepción científica e investigativa

Teniendo en cuenta la estructura interna de este tipo de competencia y los procedimientos didácticos que se proponen para su desarrollo, los principales trabajos que se han abordados sobre este tema son: Connor (1972), Quigley y Kuhne (1996), Hakkarainen, Lipponen, Jarvela y Niemivirta (1999), Rodríguez (2000), Irigoin y Vargas (2002), Morin (2002), Glass, Vessey y Ramesh (2002), Gallardo (2003), Fernández, Llivina, Arencibia, Hernández y Castellanos (2003), Pla (2005), Delamare Le Deist y Winterton (2005), Burke, Schlenk, Sereika, Cohen, Happ y Dorman (2005), Santos (2005), Cejas (2006), Arbeláez, Orlando e Ilce (2006), Muñoz, Quintero y Munévar (2006), Tobón, Rial, Ángel y García (2006), Facundo, Patricia, Carlos, Ruiz, Claro y Vanegas (2007), Velásquez (2007), Proyecto TuningAmérica Latina (2007), Gray (2007), Maldonado, Landazábal y Hernández (2007), Barnett (2008), Castillo (2008, 2011), Balbo (2008), Tobón (2008a) y Tobón (2008b), Chu, Chow, Tse y Kuhlthau (2008), Gayol, Montenegro, Tarrés y D’Ottavio (2008), Gómez (2009), Contreras (2010), Sánchez (2010), Hsing-fen, Miozzoa y Laredob 

DE LAS HABILIDADES INVESTIGATIVAS

(2010), Ortega y Jaik (2010), Association pour l'emploi des cadres [France], Deloitte [France] (2010), Bolgzda y Olehnovica (2012), Baltrūna (2013) y Bezrukova y Bezrukov (2013).

Lo antes expuesto evidencia que existen diversos enfoques para la formación de la habilidades de la competencia investigativa, su efectividad depende de las concepciones pedagógicas del investigador y el contexto histórico-social en que se desarrolla la formación, aun así las propuestas establecidas hasta el momento no se adecuan a las exigencias de las carreras con perfil en el área de la informática asociada al desarrollo de software industrial.

Los y las estudiantes de carreras profesionales con perfil informático, generalmente como parte de su proceso de formación, se incorporan a laborar en diversos centros de trabajos que les permiten contribuir a desarrollar sus competencias profesionales. Uno de estos centros son empresas o compañías que su objeto socioeconómico es la industria del software.

Desde la relación Universidad-Industria o Universidad-Empresa (existen diferentes acepciones de este tipo relación, en este trabajo se asume la primera), se debe garantizar que la labor que realicen los y las estudiantes en la industria, y en específico en el desarrollo de software industrial, contribuya a la formación de las competencias profesionales inherentes a esta profesión, y dentro de estas, la investigativa. Solo se lograría este fin si la actividad se realiza de forma intencionada, formativa y didáctica, por lo que el accionar de los responsables de la formación de estos estudiantes en el puesto de trabajo es de vital importancia.

La principal diferencia existente entre la relación Universidad-Industria y Universidad-Empresa se encuentra determinada por la diferencia estructural y organizativa existente en una empresa o una industria y, a su vez, las habilidades y competencias que desarrollan los y las estudiantes universitarios que se encuentran incorporados a estos ámbitos de trabajo son diferentes en cada contexto laboral debido a sus particularidades.

Tomando en consideración a Expósito y Díaz (2010) y Fariñas y Hurtado (2012) no se ha declarado en las Ciencias Pedagógicas ni en la Didáctica, el objeto de estudio de la disciplina de Ingeniería del Software, Base de Datos, Técnicas de Programación, entre otras que se imparten en carreras universitarias como la Ingeniería informática y otras similares; solo se ha afirmado la existencia teórica de la Didáctica de la Informática, como didáctica especial o específica, la cual tiene como objeto de estudio las regularidades del proceso de docente educativo en el marco de la enseñanza de la Informática, es decir, estudia cómo dirigir la formación de conocimientos, habilidades y valores informáticos que comprende la Informática Escolar.

La Didáctica de la Informática como didáctica específica ha sido poco investigada, los principales aportes han tenido mayor énfasis en el proceso de enseñanza-aprendizaje de la programación (Expósito y Díaz, 2010) y, en cuanto a la formación de la habilidades de la competencia investigativa asociada al desarrollo de software, han sido abordados principalmente en el componente académico 
desde el aprendizaje colaborativo y su aplicación en el aprendizaje por proyectos desde diferentes asignaturas como la ingeniería del software, la seguridad informática, la programación, base de datos y la metodología de la investigación científica, pero en el componente laboral han sido muy limitados, según las particularidades y exigencias del desarrollo de software (MINED, 1996; Díaz, 2008; Expósito y Díaz, 2010; Fariñas y Hurtado, 2012 y Expósito, 2013).

Lo antes expresado permitió determinar como problema de esta investigación: ¿Cuáles son las exigencias didácticas que se deben tener en cuenta para la formación de las habilidades de la competencia investigativa en los y las estudiantes que se encuentran en la actividad laboral asociada al desarrollo de software industrial?

Para brindar respuesta al problema declarado anteriormente se define objetivo: Determinar las exigencias didácticas y acciones a tener en cuenta para la formación de las habilidades de la competencia investigativa en los y las estudiantes que se encuentran en la actividad laboral asociada al desarrollo de software industrial. Estas exigencias y acciones están dirigidas a los responsables de la formación de las competencias profesionales en el estudiantado que se encuentra laborando en la industria del software.

Para la realización del trabajo se utilizó el método analítico-sintético, análisis documental, la observación científica, los cuestionarios y las entrevistas.

El trabajo brinda respuesta a las exigencias sociales, formativas y didácticas asociadas al contexto de la carrera de ingeniería en ciencias informáticas de la Universidad de las Ciencias Informáticas de Cuba y otras afines al desarrollo de software industrial. También es válido aclarar que se tuvieron en cuenta las exigencias investigativas y tecnológicas de la sociedad cubana y la industria del software en Cuba; las tendencias del desarrollo de software industrial a nivel internacional; y las exigencias curriculares de las carreras universitarias de perfil informático.

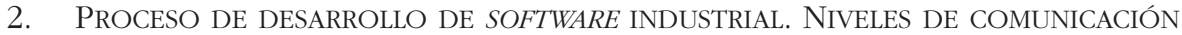

En el informe de The Association for Computing (ACM), the Association for Information Systems (AIS) and the Computer Society (IEEE-CS) (2004), se define la informática como:

Cualquier actividad que involucre las computadoras. Informática incluye: diseño y construcción de hardware y sistemas de software para cualquier propósito; procesamiento, organización y tratamiento de diversos tipos de información; estudios científicos usando computadoras; sistemas de inteligencia artificial; creación y aplicación de medios de comunicación y entretenimiento; búsqueda y recopilación de información para propósitos específicos; etc. La sociedad necesita personas que desarrollen estas tareas, por eso debemos pensar la Informática no sólo como una profesión sino también como una disciplina (p. 63). 
ODIEL ESTRADA, SAHARA MARÍA BLANCO Y FEBE ÁNGEL CIUDAD EXIGENCIAS DIDÁCTICAS EN DISEÑO DIDÁCTICO DE TAREAS PARA EL DESARROLLO DE LAS HABILIDADES INVESTIGATIVAS

2.1. ¿Dentro de la Informática a qué se le denomina proceso de desarrollo de software (PDS)?

Un PDS es aquel en que las necesidades del usuario son traducidas en requerimientos de software. Estos requerimientos son transformados en un diseño ingenieril y el diseño implementado en código, el código es probado, documentado y certificado para su uso operativo.

Ahora bien, tomando como referente a Pressman (2010) el software posee características que son únicas en correspondencia con otros elementos ingenieriles ya que este:

1. Se desarrolla, no se fabrica, esto viene dado porque los costes y los procesos se encuentran en el diseño ingenieril, no en el proceso industrial.

2. Posee un mantenimiento complejo, esto implica que la inclusión de nuevos requisitos funcionales por el cliente o el usuario final o la corrección de errores ingenieriles implica un rediseño en los artefactos ingenieriles y por consecuente en toda su arquitectura en dependencia de la magnitud del cambio realizado, provocando esto que su mantenimiento sea complejo y difícil en el área de la ingeniería, debido a que no se trabajan o se manipulan elementos físicos sino virtuales.

3. Se desarrolla a la medida, no siempre el software que se desarrolla es aplicable a cualquier usuario ya que en ocasiones se desarrolla para usuarios y clientes específicos y a la medida exigidas por estos, es decir, con fines determinados.

\section{2. ¿Cuáles son las particularidades comunicativas del PDS?}

En el PDS que se desarrolla en un ámbito industrial se evidencia como regularidad que en el grupo de desarrollo de software existen tres niveles de comunicación entre el sujeto y el grupo, estos son: comunicación entre todos los subgrupos que integran al grupo de desarrollo de software de forma sistémica; comunicación entre sujeto-grupo del rol profesional al cual pertenece el sujeto; y comunicación entre sujeto-sujeto perteneciente a otro u otros grupos con roles profesionales diferentes (ver Figura 1). 


\section{FIGURA 1}

Niveles de comunicación existentes en el Grupo de Desarrollo de Software que se evidencia en la industria del software

Grupo de desarrollo de Software

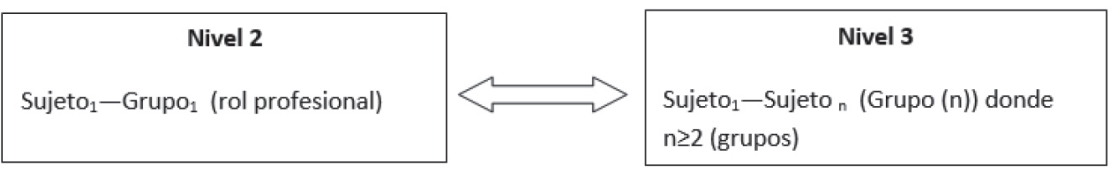

Nivel 1. Comunicación entre todos los subgrupos que integran el Grupo de Desarrollo de Software.

Fuente: Elaboración propia.

Si se desea formar en los y las estudiantes competencias profesionales acordes a las exigencias de la industria del software, lo antes expuesto evidencia que desde la didáctica de la informática se deben tener en cuenta estos niveles de comunicación.

Tomando en cuenta las particularidades comunicativas del PDS: ¿cuáles son las habilidades investigativas que deben dominar los y las estudiantes de la carrera de ingeniería en ciencias informáticas y de otras carreras afines a la informática, en correspondencia a la actividad laboral asociada al desarrollo de software industrial?

Esta actividad en cada país posee exigencias investigativas y tecnológicas distintas por lo que para responder esta pregunta, en nuestra consideración, se debe tener en cuenta el contexto histórico-social en que el estudiantado se encuentra laborando. En nuestro caso se asume la industria del software de Cuba con sus particularidades aunque también se hace necesario analizar esta industria a nivel internacional, para que la propuesta pueda tener a su vez una posible generalización.

Para identificar las habilidades investigativas que se deben desarrollar en correspondencia con el proceso de desarrollo de software industrial, se realizó:

1. Una sistematización teórica de cuáles son las habilidades investigativas que se evidencian en los roles profesionales que generalmente desempeñan los y las estudiantes cubanas de la carrera de ingeniería en ciencias informáticas y carreras afines a la informática, los roles que se analizaron fueron: analista de software, implementador (desarrollador), administrador de base de datos, arquitecto de software, asegurador de la calidad y revisor técnico. Posteriormente se determinó cuáles son las habilidades investigativas que como regularidad estaban presentes en todos los roles. 
ODIEL ESTRADA, SAHARA MARÍA BLANCO Y FEBE ÁNGEL CIUDAD EXIGENCIAS DIDÁCTICAS EN DISEÑO DIDÁCTICO DE TAREAS PARA EL DESARROLLO DE LAS HABILIDADES INVESTIGATIVAS

2. Un análisis teórico de cuáles son las habilidades investigativas asociadas a la investigación tecnológica, ya que el desarrollo de software industrial responde a este tipo de investigación.

3. Determinación de las regularidades existentes en los dos puntos anteriores. Se realizó esta acción pues los y las estudiantes cubanos de estas carreras universitarias deben en su $5 .^{\circ}$ año académico desarrollar una Tesis de Diploma en la cual deben presentar como uno de sus resultados un software. Es por ello que debe existir una relación entre las habilidades investigativas que se desarrollan en la universidad y las que se desarrollan en la industria cubana del software en donde el estudiantado está laborando.

Los referentes teóricos nacionales e internacionales que sustentaron la identificación de las habilidades que deben dominar cada uno de estos roles son Cuesta (2000), Andrés y Escobar (2005), Pérez (2007), Sommerville (2007), Blanco y Hernández (2009), Pressman (2010), Pérez (2010), Departamento Metodológico Central de Ingeniería de Software y Gestión de Software (2010), López y Andrés (2010), López, André e Infante (2011), Arza, Verdecia y Lavandero (2013) y Díaz, González y Rodríguez (2013).

El sistema de habilidades investigativas que se proponen se encuentran desglosadas por las etapas de la investigación tecnológica. Existen diferentes criterios al nombrar las etapas, en este artículo se asumen que estas son: Exploración Científica, Proyección de la investigación, Ejecución de la investigación, Análisis de los resultados del proceso investigativo, Comunicación de los resultados y Etapa de introducción y generalización de los resultados en la práctica social.

Las habilidades que se deteminaron fueron:

1. Etapa de Exploración Científica.

Habilidades:

- Comparar la realidad tecnológica actual con el modelo deseable.

- Identificar posibles problemas de investigación desde la actividad laboral.

- Formular correctamente el problema de investigación seleccionado.

2. Etapa de Proyección de la Investigación.

Habilidades:

- Determinar el objeto de la investigación.

- Determinar el campo de acción de la investigación.

- Determinar los objetivos de investigación.

- Determinar los supuestos hipotéticos (hipótesis, idea a defender o preguntas científicas).

- Determinar la población y la muestra (en el caso que proceda).

- Determinar los métodos de investigación necesarios y suficientes.

- Determinar las tareas de investigación necesaria y suficiente.

- Determinar anticipadamente los resultados de la investigación. 
ODIEL ESTRADA, SAHARA MARÍA BLANCO Y FEBE ÁNGEL CIUDAD DE LAS HABILIDADES INVESTIGATIVAS

- Determinar los tiempos necesarios para la obtención de los resultados.

- Elaborar el documento del proyecto de investigación.

- Diseñar proyectos de Tesis de Diploma.

3. Etapa de Ejecución de la Investigación.

Habilidades:

- Ejecutar las tareas planificadas.

- Ejecutar el cronograma elaborado.

- Escoger convenientemente los fundamentos teóricos que justifiquen la selección de la metodología de desarrollo de software a emplear, así como estándares, algoritmos, tecnología y herramientas informáticas a utilizar según las particularidades de la investigación a desarrollar en el área de la informática.

- Elaborar los artefactos asociados a la ingeniería de software en correspondencia a la metodología de desarrollo de software empleada.

- Aplicar normas de calidad, estándares y patrones en el proceso de desarrollo de software, según las particularidades de la investigación y de la solución que se propone.

- Aplicar métodos o procedimientos de desarrollo e implementación de software.

4. Etapa de análisis de los resultados del proceso investigativo.

Habilidades:

- Procesar los datos empíricos (categorizar, codificar, tabular y ordenar en tablas y gráficos).

- Aplicar técnicas y métodos que provee la ingeniería de software para el diseño, evaluación y análisis de la calidad del producto informático o de la solución informática implementada acorde a las características de la metodología de desarrollo de software utilizada o de los patrones, estilos y estándares ingenieriles aplicados.

- Realizar pruebas ingenieriles para la validación del software o la aplicación de métodos experimentales industriales para validar el resultado obtenido.

- Validar la hipótesis, idea a defender o el cumplimiento del objetivo de la tesis con la utilización de métodos estadísticos.

- Análizar y comparar las soluciones existentes y la solución informática que se obtuvo.

- Formular conclusiones.

- Formular recomendaciones.

5. Etapa de Comunicación de los resultados.

Habilidades:

- Presentar de forma escrita, con un lenguaje claro y accesible, los resultados de la actividad científico-investigativa. 
ODIEL ESTRADA, SAHARA MARÍA BLANCO Y FEBE ÁNGEL CIUDAD EXIGENCIAS DIDÁCTICAS EN DISEÑO DIDÁCTICO DE TAREAS PARA EL DESARROLLO DE LAS HABILIDADES INVESTIGATIVAS

- Exponer oralmente en eventos científicos los resultados de la investigación.

- Intercambiar en talleres los resultados obtenidos con otros estudiantes.

- Elaborar el Trabajo de Diploma.

- Elaborar un informe técnico donde se presenten los resultados de la investigación en la Jornada Científica Estudiantil establecido por las instituciones univesitarias cubanas.

6. Etapa de introducción y generalización de los resultados en la práctica social.

Habilidades:

- Acreditar la propiedad intelectual de los resultados obtenidos (Centro Nacional de Derecho de Autor, CENDA, si es que los resultados obtenidos proceden según las normativas del CENDA) (OPCIONAL).

- Elaborar acciones y exigencias para la introducción de los resultados obtenidos desde el despliegue de la aplicación.

Estas habilidades que se determinaron no solo van a ser utilizadas por los encargados de dirigir el proceso de formación de competencias profesionales en el estudiantado que se encuentra laborando (incorporado) en la actividad laboral vinculada al desarrollo de software industrial, sino que también son las que se van a tener en cuenta para evaluar la calidad de las tesis de diploma, garantizando en cierta medida una relación entre la universidad-industria (software).

Una vez determinadas cuáles son las habilidades investigativas, se hace necesario establecer las exigencias didácticas para su desarrollo, tomando como criterio de partida las particularidades de la industria del software.

A continuación se presentan las exigencias didácticas a tener en cuenta en la elaboración y evaluación de las tareas productivas que le son orientadas al estudiantado en la industria del software.

\section{EXIGENCIAS DIDÁCTICAS EN LA ELABORACIÓN Y EVALUACIÓN DE TAREAS}

En el proceso de orientación y evaluación de las tareas productivas se deben tener presentes por los encargados de guiar y dirigir la formación del estudiantado desde la actividad laboral vinculada al desarrollo de software industrial (tutor) las siguientes exigencias didácticas:

3.1. Nivel de comunicación entre los tres niveles que se establecen en el grupo de desarrollo de software industrial abordado anteriormente

En la orientación de la tarea productiva se necesita determinar cuáles son los niveles comunicativos de esta tarea, para ello el tutor tendría que tener en cuenta las siguientes preguntas: ¿cómo se elabora o desarrolla el artefacto que se desea 
construir con los demás artefactos que se están desarrollando en otros roles? ¿Cómo se integra el artefacto que se desea desarrollar con la arquitectura del sistema? ¿Cuáles son los niveles de interacción entre el alumno que va a resolver esta tarea y los demás integrantes de su rol y de otros roles que pertenecen a subgrupos diferentes?

En la evaluación, se debe tener en cuenta no solo la calidad del resultado ingenieril obtenido, sino además cómo se realizó, es decir, el proceso, para ello se hace necesario constatar que se cumplió con todas las exigencias presentes en la orientación de la tarea asociada a los niveles de comunicación.

\subsection{Determinación de los métodos y/o técnicas investigativas e ingenieriles a utilizar para realizar la tarea}

En la orientación se debe determinar cómo utilizar de forma integrada los métodos y técnicas investigativas con los métodos, procedimientos y/o metodologías de la ingeniería del software.

En la evaluación, el estudiante debe explicar cómo hizo uso de los métodos y técnicas investigativas en la aplicación de los procedimientos y/o metodologías ingenieriles, ya que el propósito no es que el estudiante sepa aplicar correctamente un método, procedimiento o una metodología ingenieril de forma reproductiva, sino que pueda brindar respuestas a preguntas como: ¿cuáles son los procedimientos, métodos, estándares o algoritmos establecidos para elaborar un artefacto determinado? ¿De los existentes cuál es el idóneo según las necesidades y exigencias del software? ¿Cómo valorar la calidad del resultado que obtenga según los estándares ingenieriles nacionales e internacionales?

\subsection{Explicación de la importancia de la tarea a realizar y de su repercusión en el desarrollo del software (motivación)}

Para desarrollar una o varias habilidades se deben tener en cuenta, a nuestra consideración, las motivaciones del estudiantado, es decir, el componente afectivo del individuo. Este componente es complejo, ya que en ocasiones es difícil determinar con exactitud el estado afectivo del individuo, es por ello que se sugiere prestar especial atención a la motivación en el proceso de orientación y evaluación de la tarea productiva.

En la orientación, se debe tener en cuenta por parte del tutor una explicación al estudiante acerca de la importancia de la tarea que se le está orientando, pues se asume que el alumno(a) que se encuentra en la actividad laboral vinculada al desarrollo de software industrial, al formar parte del grupo de desarrollo, su participación es activa pues de la calidad de su trabajo depende en cierta medida la calidad del producto informático, mediado, claro está, por el trabajo multidisciplinario y la colaboración. 
ODIEL ESTRADA, SAHARA MARÍA BLANCO Y FEBE ÁNGEL CIUDAD EXIGENCIAS DIDÁCTICAS EN DISEÑO DIDÁCTICO DE TAREAS PARA EL DESARROLLO DE LAS HABILIDADES INVESTIGATIVAS

En la evaluación, el estudiante debe exponer en qué medida el resultado de la tarea ha repercutido en el desarrollo de software, contribuyendo en cierta medida a concientizar al estudiantado sobre la importancia de realizar una tarea desde una concepción investigativa e ingenieril.

Las exigencias antes propuestas pueden contribuir al desarrollo de las habilidades investigativas, aun así, se proponen acciones a llevar a cabo en la industria para lograr este fin desde una perspectiva armónica e integral.

Se propone que en la industria se debe:

\subsubsection{Crear talleres de presentación de resultados parciales}

Se propone que el grupo de desarrollo de software cree espacios en los cuales el estudiantado pueda presentar los resultados de sus trabajos ante especialistas de la producción de software. Esto permitirá que los y las estudiantes que se encuentren desempeñándose en un rol profesional puedan determinar qué están realizado sus compañeros en los demás roles y cuáles son las vías que han toman para resolver ciertas deficiencias.

En estos talleres se hace necesario exigir que el estudiante exponga sus resultados desde una concepción investigativa, determinando el problema; objetivo; las tareas que se establecieron para cumplir dicho objetivo; los métodos investigativos que se emplearon; los métodos, procedimientos, técnicas, algoritmos y estándares de calidad de la ingeniería del software utilizados (en dependencia del problema); las conclusiones; recomendaciones para mejorar el trabajo, y la repercusión económica e ingenieril de la solución propuesta. Un aspecto fundamental en estos talleres es que el estudiante demuestre una coherencia metodológica entre todos los elementos mencionados anteriormente.

En estos espacios se debe propiciar que el estudiante diagnostique y valore el proceso de desarrollo de software que se está ejecutando, logrando identificar problemas (limitantes, deficiencias o carencias) y la vía de solución desde una concepción investigativa e ingenieril.

\subsubsection{Crear talleres de presentación de resultados finales}

Se propone que el estudiante elabore un informe investigativo sobre su quehacer investigativo en el desempeño de un rol profesional determinado, además debe presentar de forma oral ante el grupo de desarrollo de software el informe elaborado.

\subsubsection{Crear talleres de tesis}

Se propone que de la misma actividad laboral que están ejerciendo los y las estudiantes en la industria, se determinen posibles temas de investigación que se puedan convertir en Tesis de Diploma. 
El estudiante debe ser capaz de determinar necesidades tecnológicas en los proyectos de desarrollo de software industrial en que esté participando, lo suficientemente complejo para que pueda convertirse en una Tesis de Diploma.

En los proyectos que posee la industria se pueden crear talleres de tesis en los cuales los y las estudiantes presenten las necesidades o problemas detectados y su posible solución, elaborando para ello el diseño teórico-metodológico de la investigación.

4. VALORACIONES DE LAS OPINIONES DE LOS Y LAS ESTUDIANTES Y DE LOS Y LAS PROFESIONALES DE LA INDUSTRIA DEL SOFTWARE ENCARGADOS DE LA FORMACIÓN DEL ESTUDIANTADO

Para determinar las valoraciones de los y las estudiantes y los y las profesionales, se definieron como indicadores: (1) el de nivel de aceptación que poseen los y las estudiantes sobre el proceso de formación que reciben de los y las profesionales de la industria; (2) nivel de conocimiento y habilidades en el diseño didáctico de tareas por parte de los y las profesionales de la industria; (3) el nivel de aceptación por parte de los y las estudiantes y de los y las profesionales acerca de la propuesta presentada en este artículo; y (4) el nivel de compromiso de los y las profesionales ante la formación del estudiante. Se aplicaron los siguientes métodos y técnicas de obtención de información:

1. Entrevistas y encuestas a 120 estudiantes de la carrera de Ingeniería en ciencias informáticas en la Universidad de las Ciencias informáticas de Cuba en el curso académico 2012-2013, con el propósito de establecer: su nivel de aceptación de la propuesta realizada y la calidad de la formación recibida por los y las profesionales de la industria del software.

2. Entrevistas y encuestas a los y las profesionales de la industria del software que dirigen el proceso de formación de competencias de los y las estudiantes encuestados, con el propósito de establecer: el dominio que estos poseen en el diseño didáctico de tareas; su nivel de compromiso en la formación del estudiantado; y su nivel de aceptación de la propuesta que se realiza. Es válido aclarar que estos profesionales pertenecen a los centros de desarrollo de software de la universidad.

3. Análisis documental; permitirá determinar el dominio didáctico que poseen los y las profesionales acerca del diseño didáctico de tareas, a través de un análisis de las tareas productivas que se están orientando y evaluando en la industria del software para la formación del estudiante.

Los resultados de la aplicación de los métodos y técnicas de obtención de información pueden ser consultados en las Gráficas 1, 2, 3, 4 y 5, respectivamente. 


\section{GRÁFICA 1}

Nivel de aceptación que poseen los y las estudiantes sobre el proceso de formación que reciben de los y las profesionales de la industria.

Gráfica de dispersión (total: 120 estudiantes, calificación de 2, 3, 4 y 5)

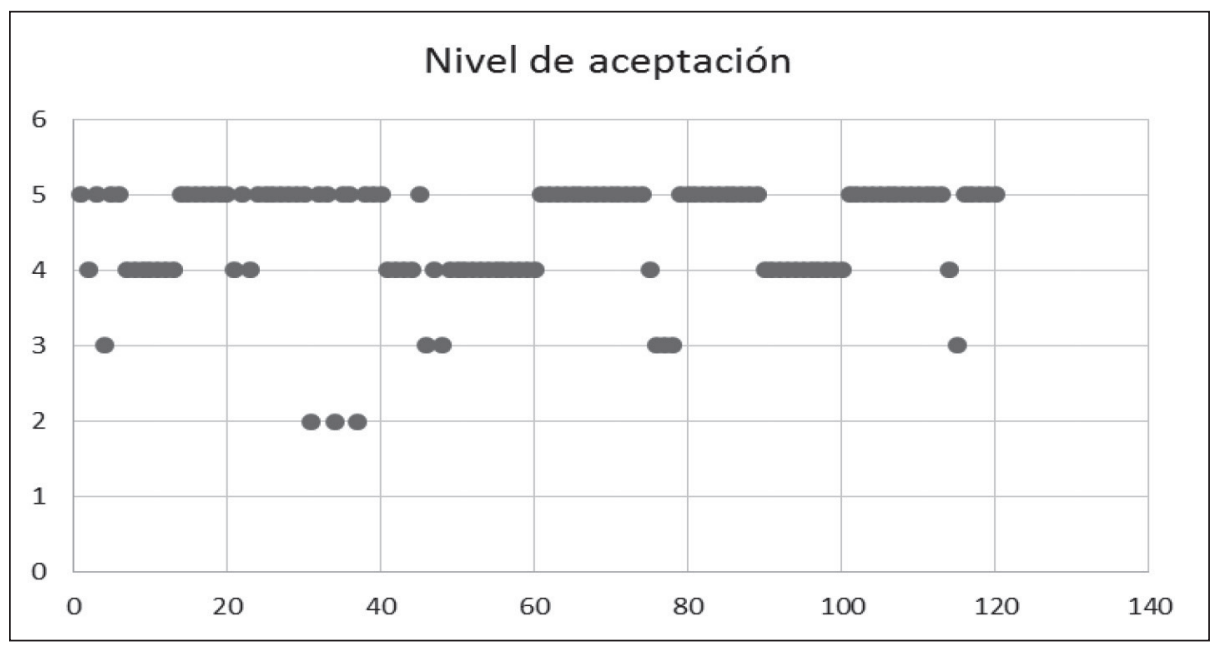

GRÁFICA 2

Nivel de conocimiento y habilidades en el diseño didáctico de tareas

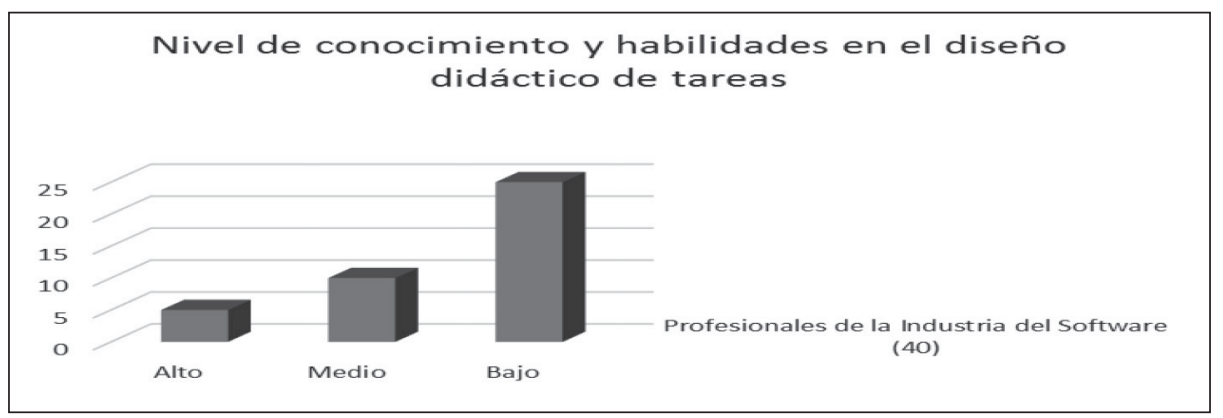


GRÁFICA 3

Nivel de aceptación por parte de los y las estudiantes acerca de la propuesta presentada en este artículo

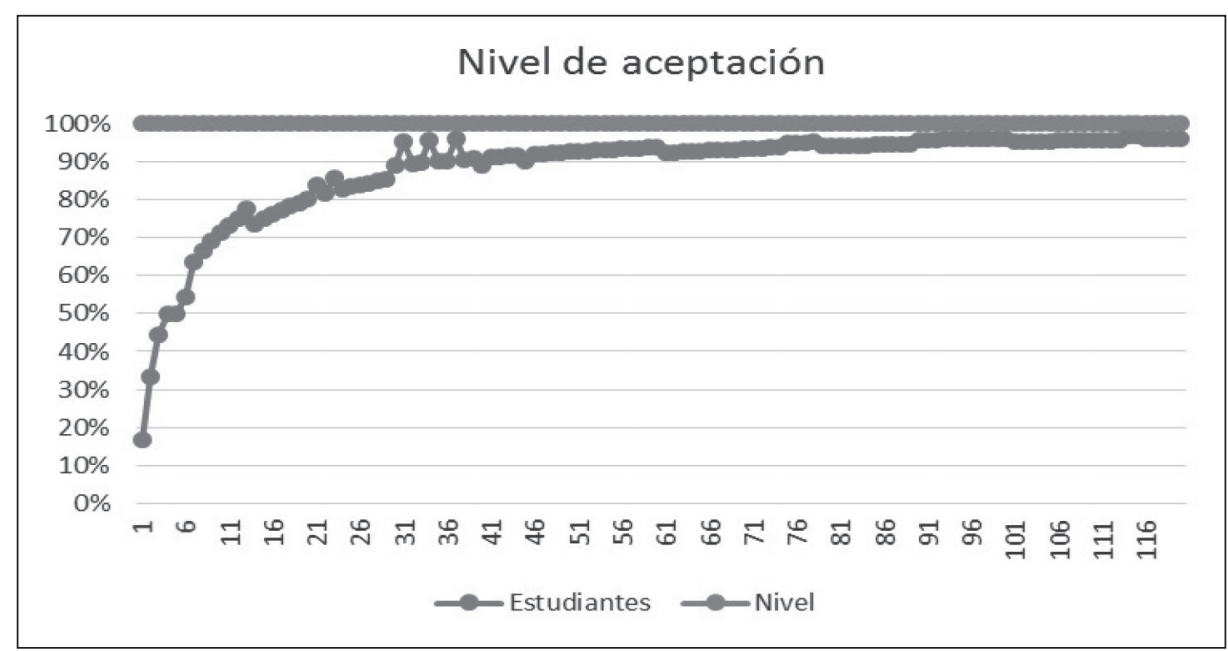

GRÁFICA 4

Nivel de aceptación por parte de los y las profesores/as acerca de la propuesta presentada en este artículo (Total de profesionales 40)

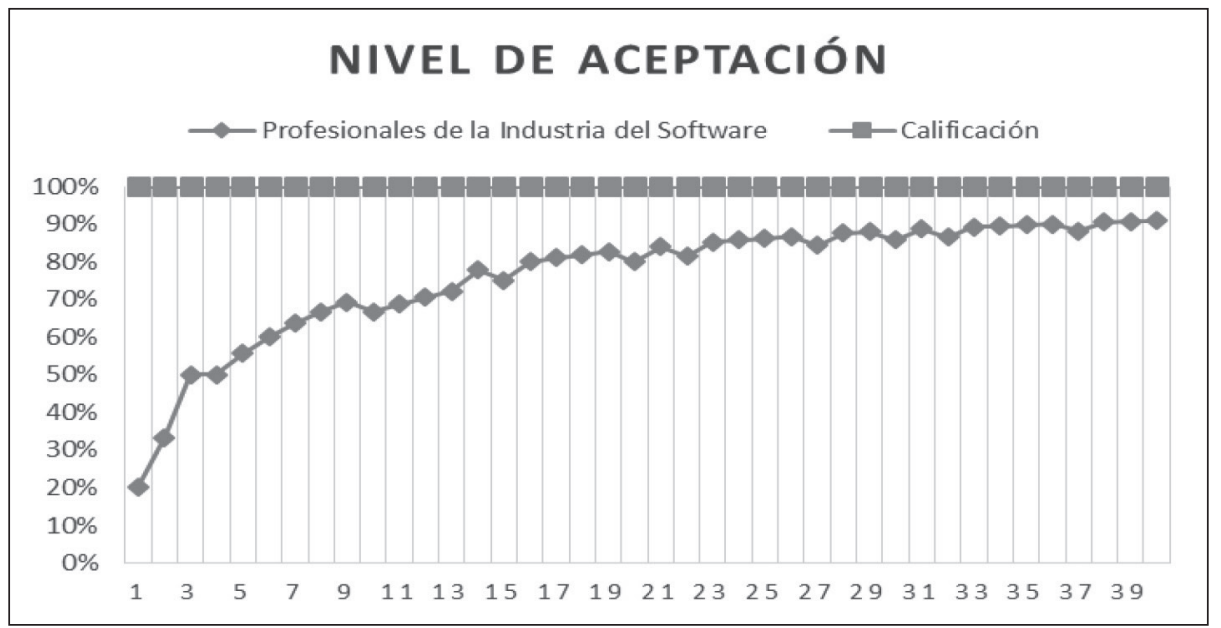



DE LAS HABILIDADES INVESTIGATIVAS

\section{GRÁFICA 5}

Nivel de compromiso de los y las profesionales ante la formación del estudiante.

Total: 40 profesionales, evaluación de 2-5, donde 5 es el máximo

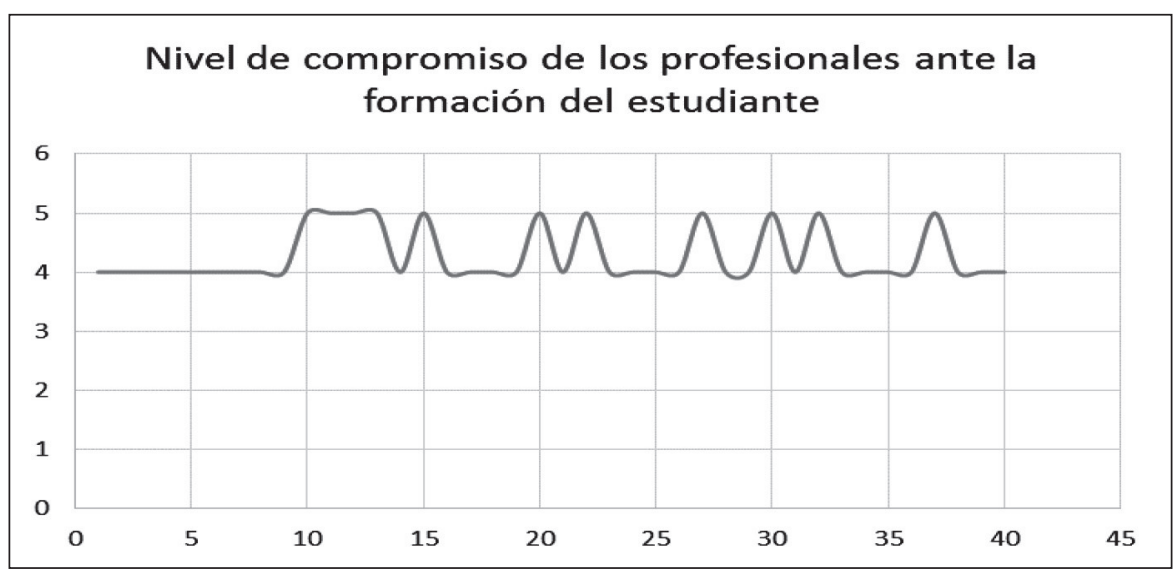

El proceso de validación y fiabilidad de las encuestas y cuestionarios realizados fue a partir de la utilización del método de expertos, para lo cual se seleccionaron 30 especialistas, 15 en Ciencias de la Educación y 15 en Ciencias Informáticas. El criterio de selección fue tomado para los casos donde exista una concordancia mayor o igual a 60\%. La cantidad de expertos se determinó a partir de la selección de una proporción del error, $\mathrm{p}=3 \%(0,03)$, un nivel de confianza $\mathrm{k}=14,84 \mathrm{y}$ un nivel de precisión de 0,12.

La representación gráfica de la información que se obtuvo en el indicador 1 evidencia que el 92\% del estudiantado opina que los y las profesionales de la industria contribuyen a su formación y que poseen altos conocimientos de ciencias informáticas y ciencias de la computación, aun así, en las entrevistas realizadas declaran que estos profesionales en el momento de orientar y evaluar las tareas productivas que les orientan solo tienen en cuenta el resultado y no el proceso de cómo se realizó.

En cuanto al indicador 2, se evidencia por parte los y las profesionales de la industria que carecen de habilidades didácticas asociadas al diseño de tareas. En el tercer indicador se evidencia el nivel de aceptación que ha tenido la propuesta didáctica que se presenta, por parte del estudiantado y de los y las profesionales, lo cual es alto.

En el cuarto indicador se evidencia que los y las profesionales de la industria están interesados en que la formación del estudiante sea eficiente, pues comprenden la implicación social, económica, tecnológica e investigativa del trabajo que puede desempeñar el estudiantado como parte del grupo de desarrollo del proyecto, además reconocen que quizás este estudiante una vez graduado se 
incorpore a laborar en este proyecto, pero se pudo constatar que los y las profesionales opinan que no son "profesores" y que su labor como director del proceso de formación es constatar solo la calidad de los resultados obtenidos por los y las estudiantes (resultado de la ejecución de las habilidades asociadas a este tipo de competencias).

Lo antes expuesto exige una necesidad educativa de concientizar a los y las profesionales de la industria del software, pues ser responsable de un proceso de formación en estudiantes universitarios requiere asumir el rol de "profesor». El desarrollo de la habilidades de la competencia investigativa en este contexto industrial conlleva no solo el desarrollo de su componente cognitivo (conocimientos y habilidades), sino a su vez el motivacional y los valores que corresponden al de un investigador.

En esta investigación se pudo determinar la necesidad de determinar exigencias didácticas que pueden guiar el trabajo de los y las profesionales de la industria, contribuyendo a estrechar las relaciones entre la universidad-industria.

Como resultado exploratorio se puede afirmar que la relación universidadindustria puede contribuir al proceso de formación del estudiante como bien se afirma en las investigaciones realizadas en los últimos cinco años, pero se debe prestar atención a cómo capacitar a los y las profesionales de la industria para que contribuyan a la formación del estudiantado desde una concepción holística, armónica y multidisciplinaria.

En el análisis teórico realizado sobre la formación de la habilidades de la competencia investigativa desde la relación universidad-industria se puede evidenciar aún una dicotomía entre los dos componentes, las soluciones actuales solo establecen las políticas educativas que establecen este vínculo o relación, pero se carece de trabajos didácticos en el componente de la industria para guiar el proceso de formación de los y las estudiantes bajo la tutela de los y las profesionales en consonancia con las ciencias pedagógicas, la didáctica, la psicología educativa y la psicología organizacional, pues son las que determinan cómo se debe formar en el individuo en un proceso de enseñanza-aprendizaje ya sea en un componente laboral o académico.

\section{CONCLUSIONES}

El desarrollo de competencias exige condiciones psicopedagógicas y didácticas acordes a un contexto educativo determinado. El desarrollo de competencias profesionales asociadas al desarrollo de software industrial requiere tener en cuenta la integración entre los niveles de comunicación existentes en este tipo de industria, el trabajo en equipos multidisciplinarios y el aprendizaje colaborativo.

La formación de las habilidades de la competencia investigativa en el contexto de la industria del software requiere que en el diseño didáctico de las tareas productivas que se le orientan al estudiante en este entorno de trabajo se tengan en cuenta la comunicación entre todos los subgrupos que integran al grupo de 
ODIEL ESTRADA, SAHARA MARÍA BLANCO Y FEBE ÁNGEL CIUDAD EXIGENCIAS DIDÁCTICAS EN DISEÑO DIDÁCTICO DE TAREAS PARA EL DESARROLLO DE LAS HABILIDADES INVESTIGATIVAS

desarrollo de software de forma sistémica; comunicación entre sujeto-grupo del rol profesional al cual pertenece el sujeto; y comunicación entre sujeto-sujeto perteneciente a otro u otros grupos con roles profesionales diferentes.

Una de las formas de favorecer el desarrollo de las habilidades de la competencia investigativa asociada a la industria del software desde la relación Universidad-Industria (Software) es establecer un sistema de habilidades que se correspondan a la investigación tecnológica, las cuales se desarrollen de forma integrada desde la concepción de esta relación. En esta investigación se encontraron exigencias didácticas y acciones a tener en cuenta para el diseño didáctico de las tareas productivas que se orientan y evalúan en la industria del software en función de contribuir a este fin.

\section{REFERENCIAS BIBLIOGRÁFICAS}

Andrés, B. y Escobar, G. (2005). Arquitectura de Proyectos de ITT: Rol del Arquitecto de Software. La Habana: Félix Varela.

Arbeláez, R.; Orlando, N. e Ilce, M. (2006). El desarrollo de la competencia investigadora en los y las estudiantes de pregrado. Docencia Universitaria, 7 (1), 5-10.

Arza, L.; Verdecia, E. Y. y Lavandero, J. (2013). Metodología para ubicar estudiantes en roles del proceso de desarrollo de software. Revista de Ingeniería Industrial, 34 (3), 5-7.

Association pour l'emploi des cadres (France), Deloitte (France) (2010). Skills and competencies needed in the research field objectives 2020. Paris: APEC.

Balbo, J. (2008). Formación en competencias investigativas, un nuevo reto en las universidad. Caracas: Universidad Central de Venezuela.

Baltrūna, A. (2013). The Essence of Learning Methodological Model and its Application in Developing Tourism Students' Research Competence. The Macrotheme Review, 2 (1), 21-25.

Barnett, B. (2008). Using action-research strategies and cohort structures to ensure research competence for practitioner-scholar leaders. Journal of Research on Leadership Education, 3 (1), 10-14.

Barrón, C. (2000). La educación basada en competencias en el marco de los procesos de globalización. México: Universidad Nacional Autónoma de México.

Bezrukova, N. y Bezrukov, A. (2013). Methodological basis of development of research competence of secondary school students in the network research community. Prague-Czech Republic: $\mathrm{CBU}$ international conference on integration and innovation in science and education.

Blanco, R. y Hernández, M. (2009). Diseño de los Perfiles de Competencias para los Roles Administrador de Gestión de Configuración y Asegurador de la Calidad de Software en la Facultad 3 de la Universidad de las Ciencias Informáticas. La Habana: La Universidad de las Ciencias Informáticas.

Bolgzda, I. y Olehnovica, E. (2012). Structure of Doctoral Students' Research Competences in Sustainability Context. World Academy of Science. Engineering and Technology, (66), 1-3.

Burke, L.; Schlenk, E.; Sereika, S.; Cohen, S.; Happ, M. B. y Dorman, J. (2005). Developing Research Competence to Support Evidence-Based Practice. Journal of Professional Nursing, 21 (6), 358-363.

http://dx.doi.org/10.1016/j.profnurs.2005.10.011 
Castillo, S. (2008). Competencias investigativas desarrolladas por docentes de Matemática. ACTA SCIENTIAE. Revista de Ensino de Ciências e Matemática, 10 (2), 3-7.

Castillo, S. (2011). Evaluación de competencias investigativas. Comunicación presentada en la XII Conferencia Interamericana de Educación Matemática (CIAM 2011). RecifeBrasil.

Cejas, E. (2006). La formación por competencias laborales: proyecto de diseño curricular para el técnico en farmacia. Tesis en opción al grado científico de Doctor en Ciencias Pedagógicas (PhD). Universidad de Ciencias Pedagógicas Enrique José Varona.

Chu, S.; Chow, K.; Tse, S. y Kuhlthau, C. C. (2008). Grade 4 Students' Development of Research Skills Through Inquiry-Based Learning Projects. School Libraries Worldwide, 14 (1), 5-11.

Colectivo de Autores (2003). Metodología de la investigación educacional. Retos y polémicas actuales. La Habana: Editorial Félix Varela.

Connor, P. (1972). Scientific research competence. Two forms of collegial Judgment. The Pacific Sociological Review, 15 (3), 8-10.

Contreras, Z. D. (2010). Las competencias investigativas. Educando. República Dominicana: Ministerio de Educación de la República Dominicana.

Cuesta, A. (2000). Gestión por Competencias. La Habana: Facultad de Ingeniería Industrial. Centro Universitario José Antonio Hecheverría de la Habana (CUJAE).

Delamare Le Deist, F. y Winterton, J. (2005). What Is Competence? Human Resource Development International, 8 (1), 27-46.

Departamento Metodológico Central de Ingeniería de Software y Gestión de Software. (2010). Perfil de competencias genéricas y específicas. La Habana: Universidad de las Ciencias Informáticas.

Díaz, G. (2008). Las tecnologías informáticas como recurso didáctico en la escuela. Comunicación presentada en el Congreso Internacional de Didáctica de las Ciencias 2008. La Habana, Cuba.

Díaz, D.; González, W. y Rodríguez, R. (2013). Métrica para establecer el progreso durante desarrollos de software. Avanzada Cientifica, 16 (2), 6-9.

Expósito, C. (2013). Entrevista realizada al doctor Expósito, metodólogo de la disciplina Informática Educativa en el Ministerio de Educación de Cuba. La Habana: Organismo Central del Ministerio de Educación. Septiembre de 2013.

Expósito, C. y Díaz, G. (2010). Informática educativa y didáctica de la informática. Comunicación presentada en el VI Congreso Internacional de Didáctica de las Ciencias. La Habana, Cuba.

Facundo, L.; Patricia, D.; Carlos, J.; Ruiz, Y.; Claro, A. y Vanegas, H. (2007). Visibilidad y formación en investigación. Estrategias para el desarrollo de competencias investigativas. STUDIOSITAS, 2 (2), 10-12.

Fariñas, J. L. y Hurtado, F. (2012). Tendencias constatadas en la praxis de la investigación pedagógica ante la selección del objeto de estudio. Revista Didasc@lia: Didáctica y Educación, 3 (6), 3-5.

Fernández, A. M.; Llivina, M. J.; Arencibia, V.; Hernández, R. y Castellanos, B. (2003). Informe técnico del resultado: Esquema conceptual, referencial y operativo (ECRO) sobre la investigación educativa. La Habana: Universidad de Ciencias Pedagógicas José Enrique Varona.

Gallardo, O. (2003). Modelo por competencias para investigadores. Contexto e Educação, $18(70), 9-25$. 

DE LAS HABILIDADES INVESTIGATIVAS

Gayol, M. D.; Montenegro, S. M.; Tarrés, M. C. y D'Ottavio, A. E. (2008). Competencias Investigativas. Su desarrollo en carreras del Área de la Salud. UNI-PLURI/VERSIDAD, 8 (8), 5-8.

Glass, R.; Vessey, I. y Ramesh, V. (2002). Research in software engineering: an analysis of the literature. Information and software technology, 44 (4), 491-506.

Gómez, D. E. (2009). Estrategia pedagógica para el desarrollo de la habilidades de la competencia investigativa en la formación del Bachiller Técnico en Alimentos. Tesis en opción al grado científico de Doctor en Ciencias Pedagógicas (PhD). Universidad de Ciencias Pedagógicas Héctor Zaldívar Pineda.

Gray, C. (2007). Research competencies framework. England: Faculty of General Dental Practice (UK). The Royal College of Surgeons of England.

Hakkarainen, K.; Lipponen, L.; Jarvela, S. y Niemivirta, M. (1999). The interaction of motivational orientation and knowledge-seeking inquiry in computer-supported collaborative learning. Journal of Educational Computing Research, 21 (3), 263-281.

Hsing-fen, L.; Miozzoa, M. y Laredob, P. P. (2010). Career patterns and competences of PhDs in science and engineering in the knowledge economy: The case of graduates from a UK research-based university. Research Policy, 39 (7), 869-878.

Irigoin, M. y Vargas, F. (2002). La formación basada en competencias. El diseño de la formación basada en competencias. Montevideo: CINTERFOR-OPS.

Irigoyen, J. J.; Yerith, M. y Karla, F. (2011). Competencias y educación superior. Revista Mexicana de Investigación Educativa, 16 (48), 244.

López, Y. y Andrés, M. (2010). Roles en el proceso de desarrollo de software para las empresas cubanas. Ingeniería Industrial, 27 (1), 5-7.

López, Y.; André, M. e Infante, A. L. (2011). Training of roles and good practice for quality work of a computer engineer. Ingeniería Industrial, 19 (3), 382-395.

Maldonado, L. F.; Landazábal, D. P. y Hernández, J. C. (2007). Visibilidad y formación en investigación. Estrategias para el desarrollo de competencias investigativas. Revista Studiositas, 2 (2), 43-56.

MINED (1996). Modelo del Profesional de la carrera de Licenciatura en Informática y Matemática. La Habana: Ministerio de Educación de Cuba (MINED).

Morin, E. (2002). Los siete saberes necesarios para la educación del futuro. Buenos Aires: Nueva Visión.

Muñoz, J.; Quintero, J. y Munévar, R. (2006). Cómo desarrollar competencias investigativas en Educación. Bogotá: Magisterio.

Ortega, E. y Jaik, A. (2010). Escala de evaluación de competencias investigativas. Praxis Investigativa REDIE, 2 (3), 72-80.

Pérez, A. (2010). Diseño del perfil por competencias del rol de Arquitecto de Software en la facultad 15 de la Universidad de las Ciencias Informáticas de Cuba. La Habana: Universidad de las Ciencias Informáticas.

Pérez, H. (2007). Procedimiento para la conformación de equipos de desarrollo de software para la Facultad 3 de la Universidad de las Ciencias Informáticas de Cuba. La Habana: Universidad de las Ciencias Informáticas.

Pérez, N. E. y Setien, E. (2008). La interdisciplinariedad y la transdisciplinariedad en las ciencias: una mirada a la teoría bibliológico-informativa. Revistas Cubanas Médicas, $18(4), 2-3$.

Pla, R. (2005). Las competencias profesionales para el desempeño del docente en la educación de los alumnos desde un enfoque integrador. La Habana: Congreso Internacional Pedagogía 2005. 
Pressman, R. (2010). Software Engineering: A practitioner's approach. 7. ${ }^{a} \mathrm{ed}$. New York: Ed. McGraw-Hill.

Proyecto Tuning-América Latina (2007). Reflexiones y perspectivas de la Educación Superior en América Latina. Informe Final del Proyecto Tuning-América Latina. Bilbao: Publicaciones de la Universidad de Deusto.

Quigley, B. y Kuhne, G. (1996). Creating practical knowledge through action research: Posing problems, solving problems, and improving daily practice. New Directions for Adult and Continuing Education (73), 7-10.

Rodríguez, M. (2000). Formación reflexiva-creativa de competencias investigativas en los docentes. Buenos Aires: CLAcso.

Rubinstein, S. (1986). El problema de las capacidades y las cuestiones relativas a la teoría psicológica. La Habana: Editorial Pueblo y Educación.

Sánchez, P. T. (2010). El proceso de formación investigativa del profesional ingeniero y la(s) competencia(s) investigativa. Pedagogía Universitaria, 15 (4), 7-10.

Santos, J. (2005). Modelo pedagógico para el mejoramiento del desempeño pedagógico profesional de los profesores de Agronomía de los Institutos Politécnicos Agropecuarios. Tesis en opción al grado científico en Doctor en Ciencias Pedagógicas (PhD). La Habana: Instituto Superior Pedagógico Enrique José Varona.

Shaw, M. (2002). What Makes Good Research in Software Engineering? International Journal of Software Tools for Technology Transfer, 4 (1), 5.

http://dx.doi.org/10.1007/s10009-002-0083-4

Sommerville, I. (2007). Software Engineering. 8. ${ }^{a}$ ed. New York: Ed. Addison-Wesley.

The Association for Computing (ACM), the Association for Information Systems (AIS) and the Computer Society (IEEE-CS). Computing Curricula 2004. Overview Report. Washington: Association for Computing. Recuperado el 29 de marzo de 2014. http://www.acm.org/ education/Overview_Draft_11-22-04.pdf.

Tobón, S. (2008a). Gestión curricular y ciclos propedéuticos. Bogotá: Ecoe.

Tobón, S. (2008b). La formación basada en competencias de la Educación Superior. El enfoque complejo. Guadalajara: Universidad Autónoma de Guadalajara.

Tobón, S.; Rial, A.; Ángel, M. y García, J. A. (2006). Competencias, calidad y educación superior. Bogotá: Magisterio.

Velásquez, R. (2007). De esto y aquello sobre la habilidades de la competencia investigativa. Avances Investigación en Ingenierías, (7), 4-7. 